\title{
Performance of an achromatic focal plane mask for exoplanet imaging coronagraphy
}

\author{
Kevin Newman ${ }^{\mathrm{a}, \mathrm{b} *}$, Ruslan Belikov ${ }^{\mathrm{b}}$, Eugene Pluzhnik ${ }^{\mathrm{b}}$, Kunjithapatham Balasubramanian ${ }^{\mathrm{c}}$, Dan \\ Wilson ${ }^{\mathrm{c}}$ \\ ${ }^{a}$ University of Arizona, Tucson, AZ \\ ${ }^{b}$ NASA Ames Research Center, Moffett Field, CA \\ 'NASA Jet Propulsion Laboratory, Pasadena, CA
}

\begin{abstract}
Coronagraph technology combined with wavefront control is close to achieving the contrast and inner working angle requirements in the lab necessary to observe the faint signal of an Earth-like exoplanet in monochromatic light. An important remaining technological challenge is to achieve high contrast in broadband light. Coronagraph bandwidth is largely limited by chromaticity of the focal plane mask, which is responsible for blocking the stellar PSF. The size of a stellar PSF scales linearly with wavelength; ideally, the size of the focal plane mask would also scale with wavelength. A conventional hard-edge focal plane mask has a fixed size, normally sized for the longest wavelength in the observational band to avoid starlight leakage. The conventional mask is oversized for shorter wavelengths and blocks useful discovery space. Recently we presented a solution to the size chromaticity challenge with a focal plane mask designed to scale its effective size with wavelength. In this paper, we analyze performance of the achromatic size-scaling focal plane mask within a Phase Induced Amplitude Apodization (PIAA) coronagraph.

We present results from wavefront control around the achromatic focal plane mask, and demonstrate the size-scaling effect of the mask with wavelength. The edge of the dark zone, and therefore the inner working angle of the coronagraph, scale with wavelength. The achromatic mask enables operation in a wider band of wavelengths compared with a conventional hard-edge occulter.
\end{abstract}

Keywords: Focal plane mask, Coronagraph, PIAA

\section{INTRODUCTION}

Coronagraphy is a powerful tool for directly imaging and characterizing extrasolar planets. A coronagraph operates by blocking the light from an on-axis star, so that the faint light from a nearby exoplanet can be observed. Many high performance coronagraph architectures, such as the Phase Induced Amplitude Apodization (PIAA) Coronagraph [1], utilize an opaque disk focal plane mask to block the light from the on-axis host star. The size of the focal plane mask largely determines the inner working angle of the coronagraph, the smallest angular distance relative to the star at which an exoplanet can be observed. Characteristics of the focal plane mask also contribute to the throughput and achievable contrast of the system.

The size of the focal plane mask must match the size of the stellar PSF. If the mask were undersized, it would allow starlight leakage and destroy the contrast. If the mask were oversized, it would be reduce the throughput of an observed planet, and waste space that could otherwise be used for detecting planets. A chromaticity challenge occurs because the size of the stellar PSF scales linearly with wavelength, and coronagraph systems prefer to observe in a broad spectral band. An ideal focal plane mask would scale its size as a function of wavelength to match the size of the stellar PSF. However, the size of a conventional hard-edge focal plane mask is fixed, and is usually chosen to match the size of the stellar PSF at the longest observational wavelength.

We propose a solution to the challenge of size-chromaticity of the focal plane mask based a diffractive optical filtering technique [2]. We have previously described the design of the mask along with some preliminary characterization [3]. In this paper, we provide a more detailed characterization of the mask performance, specifically within a PIAA coronagraph architecture. The most significant new results are contrast measurements taken within the Ames 
Coronagraph Experiment (ACE) testbed using speckle nulling wavefront control. In section two, we summarize the mask design. In section three, we present performance results from testing within the ACE testbed. In section four, we provide conclusions and plans for future work.

*knewman@email.arizona.edu

\section{FOCAL PLANE MASK DESIGN}

The mask is composed of three regions as shown in Figure 1. The size of the inner region matches the size of the stellar PSF at the shortest wavelength in the observational band. All of the light in this region needs to be rejected from the system, so it consists of a cone or tilted mirror structure to geometrically reject light out of the system. The starlight can be utilized for wavefront control, as described in Sing et al. [4]. The outer edge of the mask is sized to match the stellar PSF at the longest wavelength in the observational band. All light beyond the outer edge of the mask is a potential planet detection, so all light in this region is passed without change. The diffractive pattern between the inner region and the outer edge of the mask is designed so that the effective size of the mask scales with wavelength.

Cells in the diffractive region of the mask apply a phase shift that is relative to their depth. The depth of adjacent cells is designed to provide an alternating $0 \pi$ and $6 \pi$ phase shift for the shortest wavelength, and a $0 \pi$ and $5 \pi$ phase shift for the longest wavelength. After the focal plane mask, the next element in the optical path is a Lyot stop. The Lyot stop acts as a low-pass filter and forces interference between adjacent cells in the diffractive region of the mask. For the shortest wavelength, the interference is fully constructive, and the diffractive region is effectively fully transmissive. For the longest wavelength, the interference is fully destructive, and the diffractive region is effectively opaque. For all the midbandwidth wavelengths, the effective size of the mask scales almost linearly with wavelength.

The achromatic focal plane mask was manufactured at the NASA Jet Propulsion Laboratory Microdevices Laboratory, as described in Balasubramanian et al. [5]. The mask was made from PMGI (Poly methyl glutarimide) resist on Silicon with an Aluminum coating, which makes it entirely reflective. The mask was designed for an operating bandwidth of $\Delta \lambda / \lambda=20 \%$ in the visible spectrum $(530 \mathrm{~nm}$ to $636 \mathrm{~nm}$ ), and sized to match the focal plane PSF of the PIAA mirrors at the ACE laboratory.

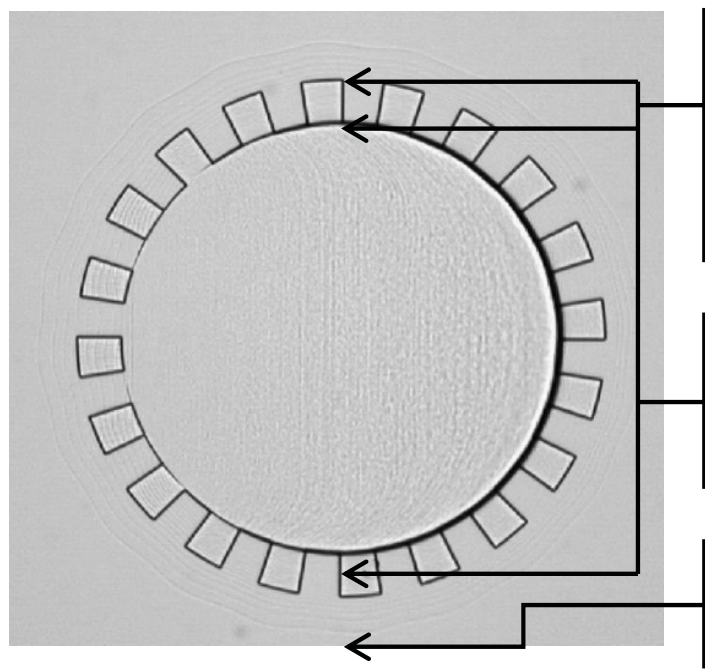

[Diffractive region]: Blocks red light from the star and planet, but allows green light from the planet to reach the science camera. Size of the stellar PSF in red light. Red light sees a grating that diffracts it out of the system while green light sees the region as a flat mirror.

[Inner region]: Rejects all light at all wavelengths. Size of the stellar PSF in green light. Center portion is tilted, so the beam is deflected outside the system.

[Outer region]: Acts as a flat mirror, reflecting all wavelengths from the planet into the science camera.

Figure 1. Microscope image of the achromatic focal plane mask fabricated at NASA JPL Microdevices Laboratory. The mask is designed with three regions. The diffractive region at the edge of the mask allows the effective size of the mask to scale approximately linearly with wavelength. 


\section{LABORATORY RESULTS}

Performance of the achromatic size-scaling focal plane mask was characterized within the ACE testbed using a PIAA coronagraph architecture. Two PIAA mirrors were used to apodize a fiber laser source. An achromatic lens re-imaged the pupil of the second PIAA mirror onto a 32 by 32 actuator deformable mirror from Boston Micromachines. The achromatic lens also focused the apodized beam onto the focal plane mask with three times magnification. The reflective focal plane mask re-directed the beam towards an undersized Lyot stop to take advantage of the diffraction effects of the mask. The beam was then imaged onto a CCD camera. We used two lasers for measurements, $532 \mathrm{~nm}$ and $630 \mathrm{~nm}$, which are very close to the minimum and maximum design bandwidth of the achromatic mask. In future testing, we plan to use a supercontinuum source to get data at intermediate wavelengths of the design bandwidth.

The plate scale at the CCD camera was calculated using Equation 1. The CCD camera had $9 m$ pixels, so the plate scale for all images with the $532 \mathrm{~nm}$ laser is 9.33 pixels per $\mathbf{b}$, and all images with the $630 \mathrm{~nm}$ laser are scaled by 11.02 a

pixels per $\boldsymbol{\omega}$.

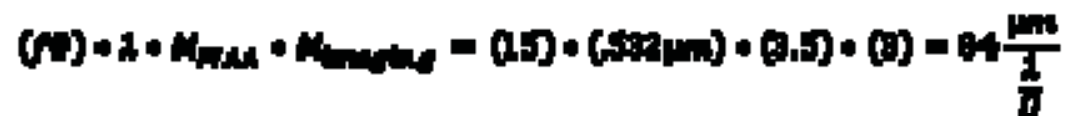

As the mask was illuminated with the $532 \mathrm{~nm}$ laser, a speckle nulling algorithm was used to create a dark hole on one side of the smallest mask $(\mathrm{D}=200 \mu \mathrm{m})$. The resulting contrast, shown in Figure 2, had mean $=2.39 \mathrm{e}-5$ and median $=$ $1.64 \mathrm{e}-5$ between 1.93 and $3.22 \lambda / \mathrm{D}$.

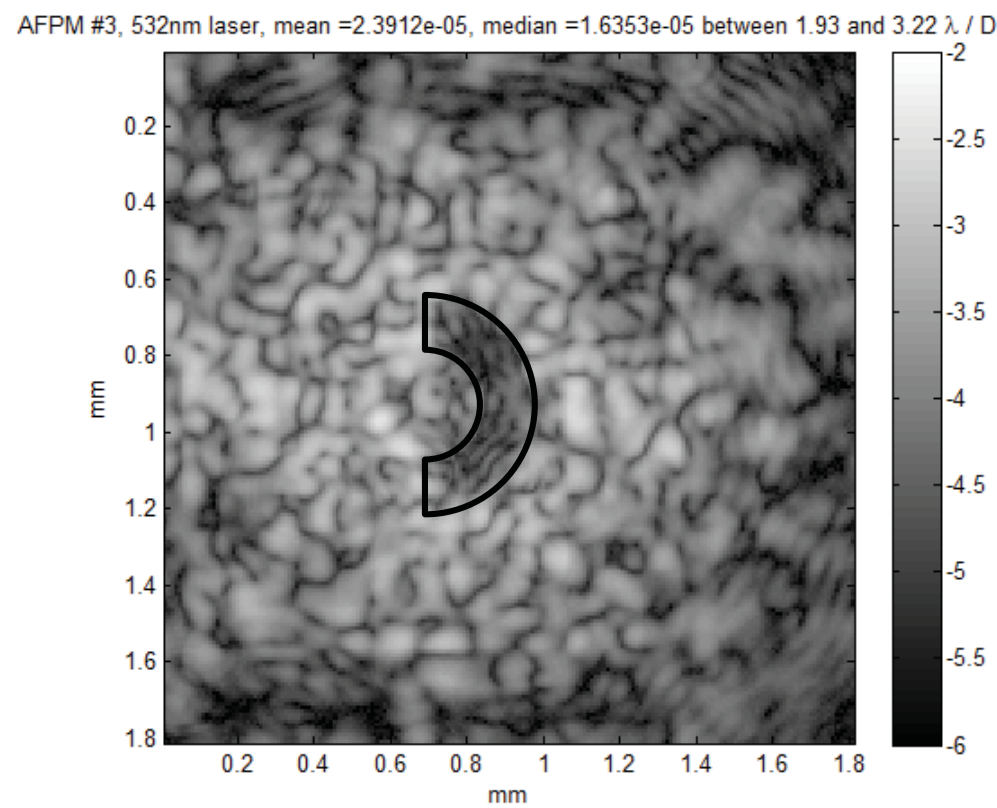

Figure 2: Contrast of an achromatic focal plane mask illuminated with an apodized PSF at 532nm. A dark hole is created on one side of the mask with a deformable mirror and a spackle nulling algorithm. 
Keeping the same pattern on the deformable mirror, we switched to the $632 \mathrm{~nm}$ laser and measured the contrast of the dark hole. The results, as shown in Figure 3, were mean $=2.83 \mathrm{e}-5$ and median $=2.19 \mathrm{e}-5$ between 1.81 and $2.90 \lambda / \mathrm{D}$. There are two important effects to note from the transition from the green to the red laser: the position of the dark hole scaled approximately with wavelength, and the contrast slightly worsened. We expect the contrast to worsen as we change wavelengths because the pattern of the deformable mirror was optimized for green illumination.

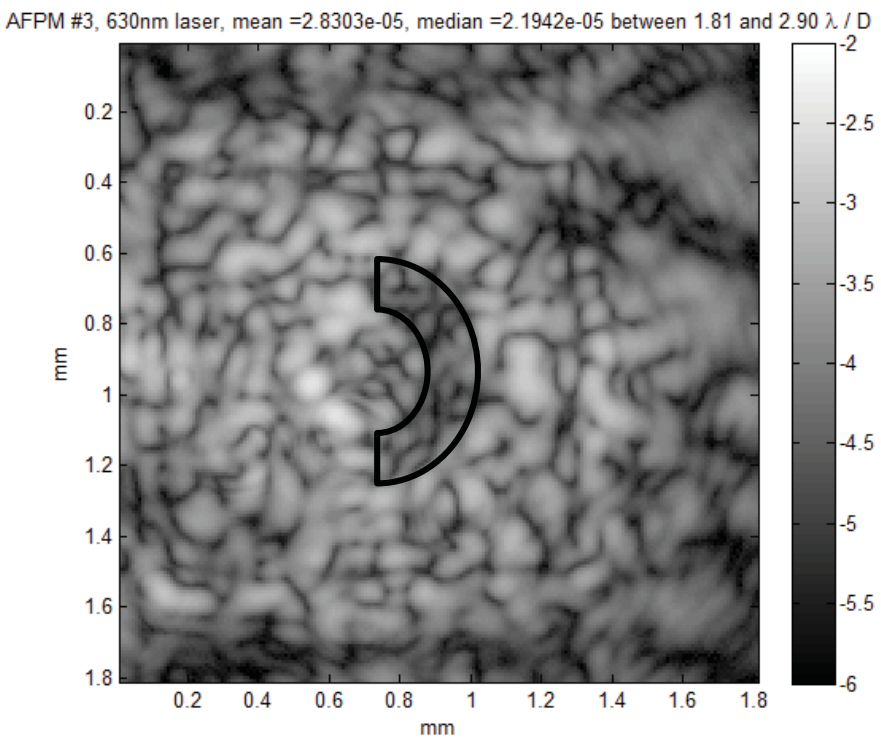

Figure 3: Contrast of an achromatic focal plane mask illuminated with an apodized PSF at 630nm. A dark hole is created on one side of the mask with a deformable mirror and a spackle nulling algorithm.

A radial intensity profile, as shown in Figure 4, shows a comparison betweeen the dark hole region at both measured wavelengths. For the profile calculation, the center of the achromatic mask is considered the starting point at zero microns of distance from the PSF center. We then examine all pixels that extend radially in the direction of the dark hole, and take an average intensity for the pixels at each radial distance from the center of the mask. The intensity values are normalized to compare the dark hole region at both measured wavelengths. The highlighted region represents the area where a planet can be discovered in green light, but would be blocked in red light to avoid starlight leaking. This concept is further explained with throughput measurements shown in Figure 5. 


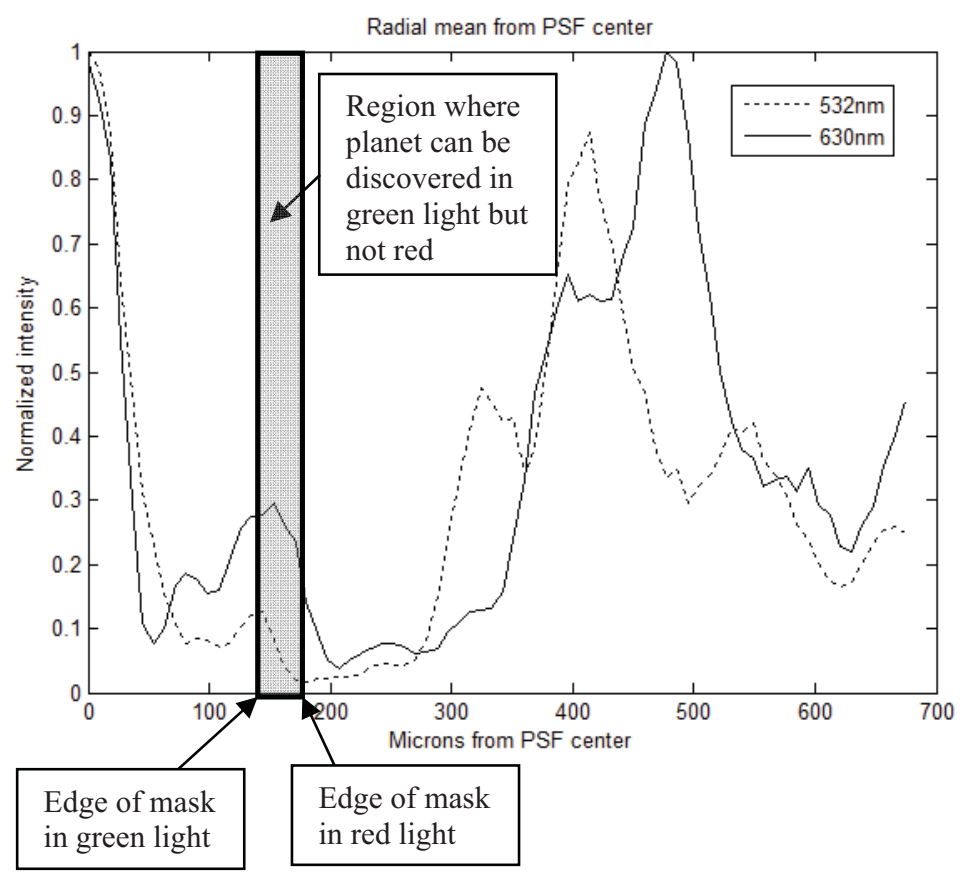

Figure 4: Intensity as a function of distance relative to the center of the achromatic focal plane mask. The location of the dark hole changes with wavelength. The intensity values on the y-axis are not normalized to full star brightness.

In another experiment using the same optical layout, we measured the throughput of an apodized PSF as a function of off-axis distance relative to the focal plane mask. For this measurement we did not use speckle nulling, and applied a flat pattern to the deformable mirror so that it behaved equivalent to a standard flat mirror. We then moved the focal plane mask relative to the apodized PSF in a single $\mathrm{x}$-direction, while taking images at each $10 \mu \mathrm{m}$ increment and calculating the total throughput of the PSF. We moved the mask relative to the PSF so that the PSF could remain on-axis, and therefore keep a symmetric shape after PIAA apodization. In future measurements, we could include an inverse PIAA system so that we could use an off-axis PSF and still recover the PSF strehl ratio. When the PSF is not entirely overlapping with the mask, the PSF represents a directly-observed exoplanet, and the total throughput is equilavent to what we could expect for an exoplanet at the same position. When compared with a conventional hard-edge focal plane mask, the achromatic mask allows for greater throughput at short wavelengths. The highlighted areas in Figure 5 show regions close to the edge of the mask where a planet could be discovered at short wavelengths, but not long wavelengths. A hard-edge mask would not be able to observe a planet in the same region at any wavelength. 


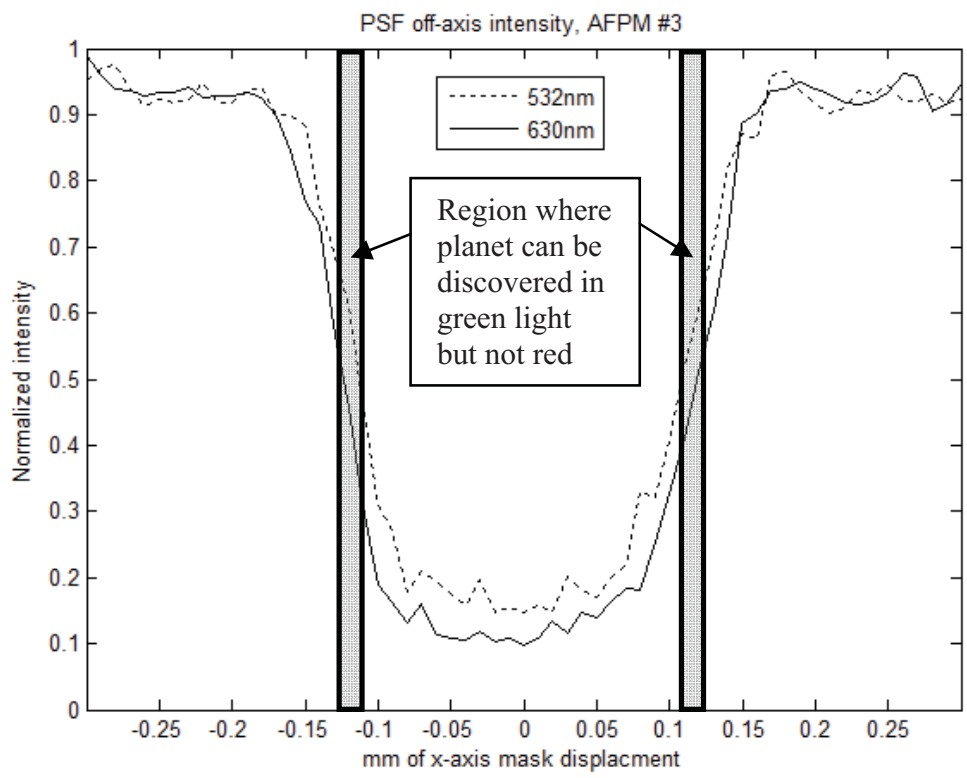

Figure 5: Throughput as a function PSF displacement relative to the center of the achromatic focal plane mask. We assume that $50 \%$ throughput is necessary for planet detection.

\section{CONCLUSIONS AND FUTURE WORK}

We have demonstrated a simple method for producing a coronagraph focal plane mask which effectively scales its size with wavelength to match the size of the stellar PSF. Performance of the mask has been characterized, with the diffractive size-scaling region of the mask operating as expected. We have demonstrated use of the mask with a PIAA coronagraph, and successful wavefront control down to a mean contrast of $2.83 \mathrm{e}-5$ between 1.81 and $2.90 \lambda / \mathrm{D}$. Speckle nulling efforts were hindered by a significant PSF drift, which could have been caused by thermal distortion in the aluminum breadboard. The mask may achieve greater contrast with improved testbed stability, and in the future we plan to take measurements using additional advanced wavefront control and a low-order wavefront sensor. We also plan to characterize the mask at intermediate wavelengths within its design bandwidth using a supercontinuum source.

An achromatic focal plane mask offers a significant advantage over a conventional hard-edge mask because the area surrounding the edge of the mask becomes available for potential planet detection at short wavelengths. The achromatic mask provides greater throughput and can be engineered to reject starlight in a manner that is favorable for wavefront sensing. The mask can be a low-risk replacement for a conventional hard-edge mask because no other components of the coronagraph need to change for the mask to operate and deliver improved coronagraph performance.

This material is based upon work supported by the National Aeronautics and Space Administration under the Astrophysics Research and Analysis (APRA) program (NASA Research Announcement NNH09ZDA001N). Any opinions, findings, and conclusions or recommendations expressed in this paper are those of the authors and do not necessarily reflect the views of the National Aeronautics and Space Administration. This work was also supported by a NASA Space Technology Research Fellowship. 


\section{REFERENCES}

[1] O. Guyon, "Phase-Induced Amplitude Apodization of Telescope Pupils for Extrasolar Terrestrial Planet Imaging", A\&A v.404, p. 379-387 (2003).

[2] R. Belikov, O. Solgaard, "Optical Wavelength Filtering by Diffraction from a Surface Relief”, Opt. Lett., Vol 28, No. 6 (2003).

[3] K. Newman, R. Belikov, O. Guyon, K. Balasubramanian, D. Wilson, "Achromatic focal plane mask for exoplanet imaging coronagraphy," Proc. SPIE Vol. 8864 (2013).

[4] G. Singh, F. Martinache, P. Baudoz, O. Guyon, T. Matsuo, N. Javonavic, C. Clergeon, "Lyot-based low order wavefront sensor for phase-mask coronagraphs: principle, simulations, and laboratory experiments," (2014).

[5] K. Balasubramanian, D. Wilson, V. White, R. Muller, M Dickie, K. Yee, R. Ruiz, S. Shaklan, E. Cady, B. Kern, R. Belikov, O. Guyon, J. Kasdin, "High contrast internal and external coronagraph masks produced by various techniques," Proc. SPIE Vol. 8864 (2013). 